\title{
Adaptive context-aware learning environments:
}

Live spaces as a basis for life-long learning in computer science

\author{
Gerald Quirchmayr and Jill Slay \\ School of Computer and Information Science, University of South Australia, Australia
}

\begin{abstract}
It is recognised that a strength of the computer science (CS) academic community has been its willingness to come to terms with the pressure on its curriculum and teaching methodology brought about by the "evolutionary" nature of the discipline (Tucker, 1996). This pressure is felt by academia in the increasing demand for just-in-time and life-long learning. Research has indicated that adaptive and interactive on-line CS learning environments play a useful role in the provision of life-long learning but these needs are not being fully met by current tools or COTS systems. This paper looks at the support of CS learning by the provision of new highly-integrated and context-aware tools.
\end{abstract}

Keywords: computer science education, life-long learning, adaptive context-aware learning environments

\section{LIFE-LONG LEARNING AND COMPUTER SCIENCE}

There is a continuing international demand for life-long learning in Computer Science (CS) and particularly, in the Australian context, increasing opportunities to develop both traditional and web-mediated joint ventures in graduate and professional education with overseas universities and multinational companies.

Some issues of concern in CS education (Tucker, 1996) in general include:

- Attrition due to poor motivation and learning difficulties.

- Dealing with students from a wide range of backgrounds with different learning styles. 
- Teaching the problem-solving and life-long learning skills demanded by industry and research.

When establishing joint cross-cultural learning projects the above issues present enormous challenges. Different researchers have examined various aspects of these issues and sought both physical and electronic means to remedy them.

\section{PHYSICAL SOLUTIONS}

\subsection{Providing motivation through active and participatory learning}

Active and participatory learning (Jenkins, 1998) are techniques that are proposed to help motivate learners. Some methods that can be used include providing opportunities in "modified lectures" for paired response to questions posed by the lecturer or students discussing the notes they have taken during the lecture and helping to correct misunderstandings. Others have used role-play to demonstrate structures and protocols for example, arrays, linked lists or token passing protocols. The problem of managing large classes complicates the issue of providing active and participatory experiences during lectures. While some feel that it is possible to manage collaborative learning in student groups of $200+$, others would reserve this type of activity for the small group or tutorial class.

\subsection{Adapting pedagogical styles to deal with social, cultural and gender issues}

Research shows that students from different cultures or of different genders display different attitudes to computers and learning.

In a two-year study of female and international CS students at Carnegie Mellon University, issues which arose included the perception by some female students that the 'purpose' of computing needed to be defined within introductory CS courses (Fisher, Margolis and Miller, 1997). While they displayed a high level of interest in the computing process they needed to be able to contextualise this process 'within a larger purpose'. They also displayed a lower level of "attachment" to their computers than did male students on the same course and expressed some relief as they discovered that CS education covered a wide range of topics.

Other research points out that there is a link between culture and learning style (Cobern, 1991; and Slay, 1999). Assertions made in this research 
indicate that Chinese students would find it easier to understand and apply theoretical principles within programming than would a similar group of Western students. In their study, Fisher, Margolis and Miller discovered that international female students on their course showed the least 'attachment' to computers or computing and used pragmatic reasoning (such as employability) for their choice of major (Fisher, Margolis and Miller, 1997).

The conclusion here is that some allowance has to be made for cultural and gender preferences within the teaching of CS. While it is possible to provide an inclusive focus within lectures, there is, however, a more pressing need to be able to adapt teaching material for different styles and preferences.

\subsection{Problem-solving for life-long learning}

It has been noted that many students who have difficulties across the first year of CS as a whole do not know where to start with a task, regardless of the subject area (Barnes, Fincher and Thompson, 1997).

Some effort has been made to incorporate training in problem-solving skills and techniques into early CS education to deal with this problem. This ranges from the use of Edward de Bono's tools for lateral thinking to the development of Polya's approach of Understand, Design and Review (Barnes, Fincher and Thompson) for problem-solving and offering courses in these techniques within, or parallel to, early programming subjects.

\section{WEB-MEDIATED SOLUTIONS}

With the problems imposed by large classes, and the large range of individual approaches needed to deal with some of the student learning issues raised above, CS academics have been some of the first to develop and use web-mediated learning environments for enhancing student learning in CS. These have been shown to provide a domain within which motivation, problem-solving, communication and flexibility can be provided.

As one of the authors has pointed out, the web provides a vehicle for the development of the learning environment and teaching can be structured to develop life-long learning skills and to provide special motivations and cater for the expectations, learning styles and demands for flexibility from students from different cultures and backgrounds (Slay, 1998a; Slay, 1998b).

Early Australian examples of this style of teaching in CS education are many. Recent Australian examples of the use of the WWW in CS education abound. Boalch provides an examination of the use of the WWW as a 
support medium for the delivery of a first year unit in information systems at Curtin University (Boalch, 1996). He provides an evaluation of site utilisation and user feedback in the case where subject information and course details were provided on the WWW for students.

The Eklunds examine the use of the WWW to supplement traditional IT teaching (Ecklund and Ecklund, 1996). They provide case studies of two examples of the re-structuring of traditional forms of IT course for webdelivery. Jones of Central Queensland University gives details of a case study involving the design, presentation and evaluation of an undergraduate unit in systems administration taught completely via the WWW to oncampus and distance students (Jones, 1996).

However, many systems that have been developed rely on the low-level concept of interactivity (derived from a distance education paradigm) as the relationship between an individual student and text, and fail to use the technological interactivity which is available. Common on-line learning environments often fail to maximise the potential of current CS research. While the value of HTML pages and threaded discussions is acknowledged, especially for those who do not have English as a first language, they do not display the ability to adapt teaching material for the needs of individual students.

\subsection{Adaptive teaching on the WWW}

Various researchers who have experimented with adaptive teaching on the WWW have used techniques and principles derived from intelligent tutoring and particularly Anderson's rule-based cognitive modelling (Anderson, 1983).

Others have used adaptive navigation support to provide adaptive navigation through hypertext pages and have thus developed adaptive textbooks for the tutoring, particularly, of software applications (Brusilovsky, 1996). While proving useful, these do not supply the full functionality of traditional ITS.

A new approach towards the support of CS education is needed. Isolated tools are of limited use in practice, and COTS systems only provide a partial solution since their integration, self-adaptation and configuration offer very little flexibility. This paper looks at the concept of live spaces and its adaptability for defining a framework that can lead to systems that can provide adaptive CS learning environments. 


\section{THE CONCEPT OF LIVE SPACES APPLIED TO CS LEARNING ENVIRONMENTS}

Use of the term 'live' in live spaces reflects the fact that the environment is dynamic (or alive) in that it changes depending on its context of use, is driven by adaptive workflow mechanisms and uses new forms of adaptive media. 'Space' reflects the notion of through-wall environments that allow participation from individuals in other locations (or spaces) by way of virtual avatars, mobile computing and extended video-conferencing. Live spaces are also an extension of workspaces, a concept that has been around for a considerable time.

The fundamental requirement is that a system has to be able to handle a range of different situations in certain contexts. It has also become increasingly unacceptable for users to have to adapt to systems instead of systems adapting to their needs. Life-long learners, often highly-qualified IT professionals, are demanding to have the necessary system capabilities accessible when and where they need them. Their teachers want to provide the adaptive and interactive content which modern CS pedagogical research is indicating.

In a CS teaching context, this means that the tools, rendering mechanism and sources of support material must be adapted to the subject (for example, AI, C++ programming, SQL) being studied. Many students are active professionals and their learning needs vary from day to day and project to project.

The most advanced concept is being pursued by DSTC, based on the Elvin enterprise bus, a notification service (see http://elvin.dstc.edu.au for Elvin details). Business processes are served by the underlying IT infrastructure via the Elvin enterprise bus, which allows services to be connected via standard interfaces. This model, which works well for enterprise models, can also serve as the basis for creating educational live spaces.

CS education has often used a traditional intelligent tutoring system, formed of two parts - an expert system and a communication module. Within the expert system, there are three modules - the student module, the pedagogical module and the domain knowledge module. The student module gives the student history; the pedagogical model provides information about the teaching process; and the domain knowledge module provides the material that is being taught. While proving useful, adaptive teaching on the WWW has not yet been able to supply the full functionality of traditional ITS although ITS researchers have fully modelled and prototyped the processes necessary for their full implementation. 
The major components of an educational live space could be grouped into sources, supplying the student history, teaching process and teaching content with attached access processes, decision processes and deliverable creation processes, with decision processes being only partly automated. Processes attached to the sources guarantee that the support environment is active (depending on whether the situation is reactive or proactive) rather than waiting for the user to push it into action.

This model of an educational live space leads to a framework for setting up context-aware CS learning environments, which are able to respond to specific needs of users.

\section{DESIGNING EDUCATIONAL LIVE SPACES ON THE WEB}

To guarantee high usability and flexibility, the best technology choice for setting up a context-aware legal work environment today is the web. Access and rendering can be assured for a wide variety of devices, ranging from mobile phones and handheld systems to powerful server and desktop environments. Depending on the end-user equipment, display capabilities might be limited, but assuming that an advanced handheld or laptop computer will be the standard, live spaces can be considered a realistic perspective. Presuming that most information is text-based, rendering power need not be excessive. Consequently, the computational power can to a large extent be concentrated in the server infrastructure feeding the system, where it will be needed for running highly complex search, scheduling and reasoning algorithms. The end-user device will, due to this high computational complexity have to be limited to the role of an I/O client with little local intelligence, but it needs a reasonable display. With most of the system intelligence being concentrated on the server, high bandwidth and stable communications become essential.

To meet the end-user demands, the server infrastructure will have to be highly configurable and will have to rely on advanced middleware, of which workflow management systems are a major component.

As this middleware is itself only a component that has to be able to communicate with other building blocks of the system, grouping the whole infrastructure around the Elvin bus (an idea first proposed by Rudi Vernik at DSTO/ITD and UniSA/ACRC in Vernik, Quirchmayr and Thomas, 2001) (see Figure 1), can be used as a starting point for a highly flexible solution.

This architecture, with system components communicating via an enterprise bus, is highly flexible and allows the concentration of computationally intensive tasks, such as searching, scheduling, and 
simulation on the servers. Limiting the client to display functionality gives the possibility to use a wide range of equipment, starting from advanced mobile phones. The only remaining restriction is the quality of the display, which in reality means that handheld computers currently are the lower boundary. For educational applications the highest workload will be on the workflow system, the knowledge and the information manager. Being able to flexibly plug in different information, knowledge management, and workflow management systems is the biggest strength of this approach. With the web becoming accessible from different types of widely spread devices, it is the obvious rendering platform for results delivered by the support environment. Access comes at a reasonable cost and can be considered as quasi-ubiquitous.

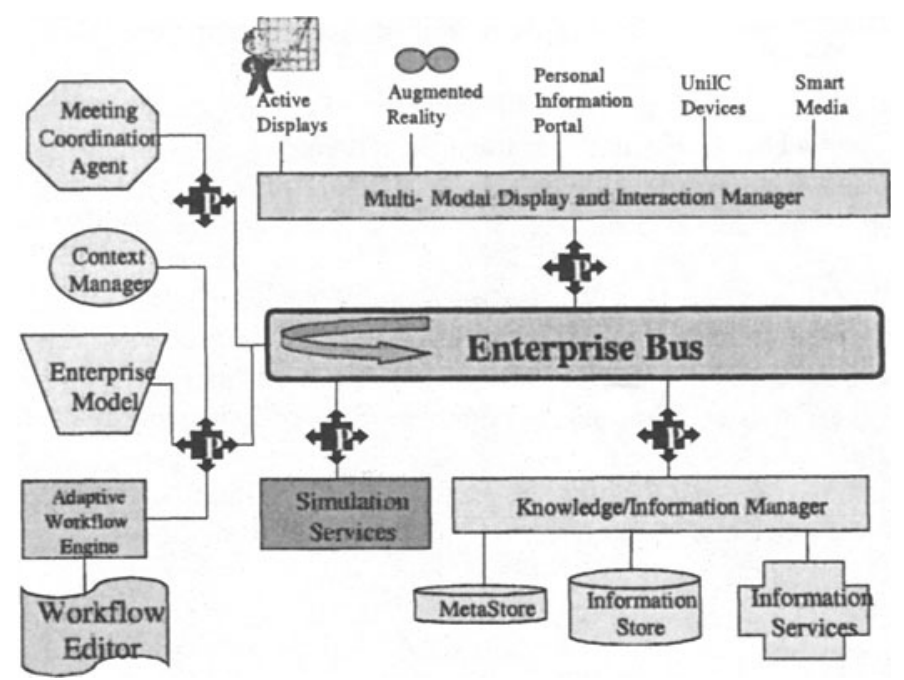

Figure 1. ACAWE Reference Architecture (Source: Vernik, Quirchmayr and Thomas, 2001)

\section{CONCLUSION}

The aim of the paper was to show how adaptive context-aware work environments could contribute to improving learning environments for computer science professionals and to suggest a way towards adapting and implementing the approach relying on a web-based delivery. With technological infrastructures becoming highly flexible due to recent developments, mainly enterprise buses, and improved web access, the time seems to be right for an attempt towards designing a new generation of computer science learning environments. 


\section{REFERENCES}

Anderson, J. (1983) The architecture of cognition. Cambridge, MA: The MIT Press

Barnes, D.J., Fincher, S. and Thompson, S. (1997) Introductory Problem-solving in Computer Science. $5^{\text {th }}$ Annual Conference on the Teaching of Computing, Dublin.

Boalch, G. (1996) WWW as an Educational Support Medium: An Australian Case Study. Paper presented at AusWeb96, Gold Coast, Australia, July 1996.

Brusilovsky, P. (1996) Integrating Hypermedia and Intelligent Tutoring Systems: From Systems to Authoring Tools. AIED.

Cobern, W.W. (1991) World view theory and science education research. NARST Monograph No. 3. Manhattan, KS: National Association for Research in Science Teaching

Ecklund, J. and Ecklund, P. (1996) Integrating the web and the teaching of technology: Cases across two universities. Paper presented at AusWeb96, Gold Coast, Australia, July 1996.

Fisher, A., Margolis, J. and Miller, F. (1997) Undergraduate Women in Computer Science: Experience, Motivation and Culture. SIGCSE, 1997.

Jenkins, T.A. (1998) Participatory Approach to teaching Programming. Dublin, Ireland: ITiCSE

Jones, D. (1996) Solving some problems of University Education: A Case Study. Paper presented at AusWeb96, Gold Coast, Australia, July 1996.

Slay, J. (1998a) Using the World Wide web to Create Foundations for Life-long Learning An Australian Perspective. Teleteaching, IFIP World Computer Congress98, Vienna, Sept 2nd 1998.

Slay, J. (1998b) Using the WWW to Create an Effective Cross-Cultural Learning Environment. Hong Kong Web Symposium 98, Hong Kong University.

Slay, J. (1999) Unpublished Ph.D. thesis. Curtin University of Technology, 1999-09-21

Tucker, A.B. (1996) Strategic Directions in Computer Science Education. ACM Computing Surveys, 28, (4)

Vernik, R., Quirchmayr G. and Thomas, B. (2001) Project proposal LiveSpaces: Adaptive Context-Aware Work Environments. University of South Australia.

\section{BIOGRAPHIES}

Jill Slay is a senior lecturer in the School of Computer and Information Science. She is currently director of international programmes within the school and manages the offshore programmes taught in Hong Kong and Malaysia. Gerald Quirchmayr holds doctoral degrees in computer science and law from Johannes Kepler University in Linz (Austria) and currently is professor at the Institute for Computer Science and Business Informatics at the University of Vienna. 